\title{
McKay Centralizer Algebras
}

\author{
Georgia Benkart $\|^{\mid}$and Tom Halverson非 \\ ${ }^{1}$ University of Wisconsin - Madison, Madison, WI 53706 USA \\ ${ }^{2}$ Macalester College, Saint Paul, MN 55105 USA
}

\begin{abstract}
For a finite subgroup $\mathrm{G}$ of the special unitary group $\mathbf{S U}_{2}$, we study the centralizer algebra $Z_{k}(\mathrm{G})=$ End $_{\mathrm{G}}\left(\mathrm{V}^{\otimes k}\right)$ of $\mathrm{G}$ acting on the $k$-fold tensor product of its defining representation $\mathrm{V}=\mathbb{C}^{2}$. The McKay correspondence relates the representation theory of these groups to an associated affine Dynkin diagram, and we use this connection to study the structure and representation theory of $Z_{k}(G)$ via the combinatorics of the Dynkin diagram. When $G$ equals the binary tetrahedral, octahedral, or icosahedral group, we exhibit remarkable connections between $\mathrm{Z}_{k}(\mathrm{G})$ and the Martin-Jones set partition algebras.

Résumé. Pour un sous-groupe fini $G$ du groupe unitaire spéciale $S U_{2}$, nous étudions la centralisateur algébre $Z_{k}(G)=$ End $_{\mathrm{G}}\left(\mathrm{V}^{\otimes k}\right)$ de $\mathrm{G}$ agissant sur le produit $k$-fold de tenseur de sa représentation définissant $\mathrm{V}=\mathbb{C}^{2}$. La correspondance de McKay concerne la théorie des représentations de ces groupes á une associé Dynkin diagramme, et nous utiliser cette connexion pour étudier la structure et la théorie des représentations de $Z_{k}(G)$ par l'intermédiaire de la combinatoire du diagramme de Dynkin. Quand $\mathrm{G}$ est égale à la groupe tétraédrique binaire, octaédre binaire, ou icosaédrique binaire, nous exhibons connexions remarquables entre $Z_{k}(G)$ et les algébres de partitions de Martin-Jones.
\end{abstract}

Keywords. Schur-Weyl duality, McKay correspondence, partition algebra, Temperley-Lieb algebra, Dyck paths

\section{Introduction}

In 1980, John McKay [13] discovered that there is a natural one-to-one correspondence between the finite subgroups of the special unitary group $\mathrm{SU}_{2}$ and the simply-laced affine Dynkin diagrams. Let $\mathrm{V}=\mathbb{C}^{2}$ be the defining representation of $\mathrm{SU}_{2}$, and let $\mathrm{G}$ be a finite subgroup of $\mathrm{SU}_{2}$ with irreducible modules $\mathrm{G}^{\lambda}, \lambda \in \Lambda(\mathrm{G})$. The representation graph $\mathcal{R}_{\mathrm{V}}(\mathrm{G})$ (also known as the McKay graph or McKay quiver) has vertices indexed by the $\lambda \in \Lambda(\mathrm{G})$ and $a_{\lambda, \mu}$ edges from $\lambda$ to $\mu$ if $\mathrm{G}^{\mu}$ occurs in $\mathrm{G}^{\lambda} \otimes \mathrm{V}$ with multiplicity $a_{\lambda, \mu}$. Almost a century earlier, Felix Klein had determined that a finite subgroup of $\mathrm{SU}_{2}$ must be one of the following: (a) a cyclic group $\mathbf{C}_{n}$ of order $n$, (b) a binary dihedral group $\mathbf{D}_{n}$ of order $4 n$, or (c) one of the 3 exceptional groups: the binary tetrahedral group $\mathbf{T}$ of order 24 , the binary octahedral group $\mathbf{O}$ of order 48, or the binary icosahedral group I of order 120. McKay's observation was that the representation graph of $\mathbf{C}_{n}, \mathbf{D}_{n}, \mathbf{T}, \mathbf{O}, \mathbf{I}$ corresponds exactly to the affine Dynkin diagram $\hat{A}_{n-1}, \hat{\mathrm{D}}_{n+2}, \hat{\mathrm{E}}_{6}, \hat{\mathrm{E}}_{7}, \hat{\mathrm{E}}_{8}$.

\footnotetext{
†Email: benkartemath.wisc.edu. G. Benkart is grateful to the Simons Foundation for the support she received as a Simons Visiting Professor at MSRI.

$\ddagger$ Email: halverson@macalester. edu. T. Halverson was partially supported by National Science Foundation grant DMS0800085 and partially supported by Simons Foundation grant 283311.
} 
In the papers [1], [2], [7], we examine the McKay correspondence from the point of view of Schur-Weyl duality. Since the McKay graph provides a way to encode the rules for tensoring by $\mathrm{V}$, it is natural to consider the $k$-fold tensor product module $\mathrm{V}^{\otimes k}$ and to study the centralizer algebra $\mathrm{Z}_{k}(\mathrm{G})=\operatorname{End}_{\mathrm{G}}(\mathrm{V} \otimes k)$ of endomorphisms that commute with the action of $G$ on $V^{\otimes k}$. The algebra $Z_{k}(G)$ provides essential information about the structure of $\mathrm{V}^{\otimes k}$ as a G-module, as the projection maps from $\mathrm{V}^{\otimes k}$ onto its irreducible $\mathrm{G}$-summands are idempotents in $Z_{k}(\mathrm{G})$, and the multiplicity of $\mathrm{G}^{\lambda}$ in $\mathrm{V}^{\otimes k}$ is the dimension of the $Z_{k}(\mathrm{G})$-irreducible module corresponding to $\lambda$.

The main points of this article are:

- The irreducible $Z_{k}(G)$-modules are labeled by vertices of the representation graph $\mathcal{R}_{\mathrm{V}}(\mathrm{G})$.

- The dimension of the $Z_{k}(\mathrm{G})$-module labeled by $\lambda$ is the number of $k$-walks from 0 to $\lambda$ on $\mathcal{R}_{\mathrm{V}}(\mathrm{G})$.

- The dimension of $Z_{k}(\mathrm{G})$ equals the number of $2 k$-walks on $\mathcal{R}_{\mathrm{V}}(\mathrm{G})$ starting and ending at 0 .

- $Z_{k}(G)$ has generators corresponding to the nodes in $\mathcal{R}_{\mathrm{V}}(\mathrm{G})$, as well as generators corresponding to each embedding $Z_{i}(G) \subseteq Z_{i+1}(G)$, and the relations are determined by the $\mathcal{R}_{V}(G)$ edge structure.

- $Z_{k}(\mathrm{G})$ has a basis of words in these generators that correspond to $2 k$-walks on $\mathcal{R}_{\mathrm{V}}(\mathrm{G})$.

- When $G$ is one of the exceptional groups $\mathbf{T}, \mathbf{O}, \mathbf{I}$, the centralizer $Z_{k}(G)$ can be described using the Martin-Jones partition algebras and their analogs.

- New formulas for the dimensions of the irreducible representations of partition algebras are given.

When $\mathrm{G}$ is a subgroup of $\mathrm{SU}_{2}$, the centralizer algebras satisfy the reverse inclusion $\mathrm{Z}_{k}\left(\mathrm{SU}_{2}\right) \subseteq \mathrm{Z}_{k}(\mathrm{G})$. It is well known that $Z_{k}\left(S_{2}\right)$ is isomorphic to the Temperley-Lieb algebra $\mathrm{TL}_{k}(2)$. Thus, the centralizer algebras constructed here all contain a Temperley-Lieb subalgebra. The dimension of $\mathrm{TL}_{k}(2)$ is the Catalan number $\mathcal{C}_{k}=\frac{1}{k+1}\left(\begin{array}{c}2 k \\ k\end{array}\right)$, which counts walks of $2 k$ steps that begin and end at 0 on the representation graph of $\mathrm{SU}_{2}$, i.e. the Dynkin diagram $\mathrm{A}_{+\infty}$. In this case, the walks correspond to Dyck paths.

\section{McKay Centralizer Algebras}

The special unitary group $\mathrm{SU}_{2}$ is the group of $2 \times 2$ complex matrices $\left(\begin{array}{cc}\alpha & \beta \\ -\bar{\beta} & \bar{\alpha}\end{array}\right)$ satisfying $\alpha \bar{\alpha}+\beta \bar{\beta}=1$. For each $r \geq 0, \mathrm{SU}_{2}$ has an irreducible module $\mathrm{V}(r)$ of dimension $r+1$. The module $\mathrm{V}=\mathrm{V}(1)=\mathbb{C}^{2}$ with basis $\mathrm{v}_{-1}=(1,0)^{\mathrm{t}}, \mathrm{v}_{1}=(0,1)^{\mathrm{t}}$ corresponds to the natural two-dimensional representation on which $\mathrm{SU}_{2}$ acts by matrix multiplication. These modules satisfy the Clebsch-Gordan formula,

$$
\mathrm{V}(r) \otimes \mathrm{V}=\mathrm{V}(r-1) \oplus \mathrm{V}(r+1),
$$

where $\mathrm{V}(-1)=0$. The representation graph $\mathcal{R}_{\mathrm{V}}\left(\mathrm{SU}_{2}\right)$ is the infinite graph shown in Figure 1

Now let $\mathrm{G}$ be a subgroup of $\mathrm{SU}_{2}$. Then $\mathrm{G}$ acts on the natural two-dimensional representation $\mathrm{V}=\mathbb{C}^{2}$ by restriction. Let $\left\{\mathrm{G}^{\lambda} \mid \lambda \in \Lambda(\mathrm{G})\right\}$ denote a complete set of pairwise non-isomorphic irreducible finitedimensional G-modules occurring in some $\mathrm{V}^{\otimes k}$ for $k=0,1, \ldots$ By convention $\mathrm{V}^{\otimes 0}=\mathrm{G}^{(0)}$ is the trivial $\mathrm{G}$-module. The representation graph $\mathcal{R}_{\mathrm{V}}(\mathrm{G})$ is the graph with vertices labeled by elements of $\Lambda(\mathrm{G})$ with $a_{\lambda, \mu}$ edges between $\lambda$ and $\mu$ if the decomposition of $\mathrm{G}^{\lambda} \otimes \mathrm{V}$ into irreducible $\mathrm{G}$-modules is given by

$$
\mathrm{G}^{\lambda} \otimes \mathrm{V}=\bigoplus_{\mu \in \Lambda(\mathrm{G})} a_{\lambda, \mu} \mathrm{G}^{\mu}
$$

For finite subgroups $\mathrm{G} \subseteq \mathrm{SU}_{2}$, the representation graph $\mathcal{R}_{\mathrm{V}}(\mathrm{G})$ is an undirected, simple graph (see [15]). Since $V$ is faithful (being the defining module for $G$ ) and $G$ is finite, all irreducible $G$-modules 


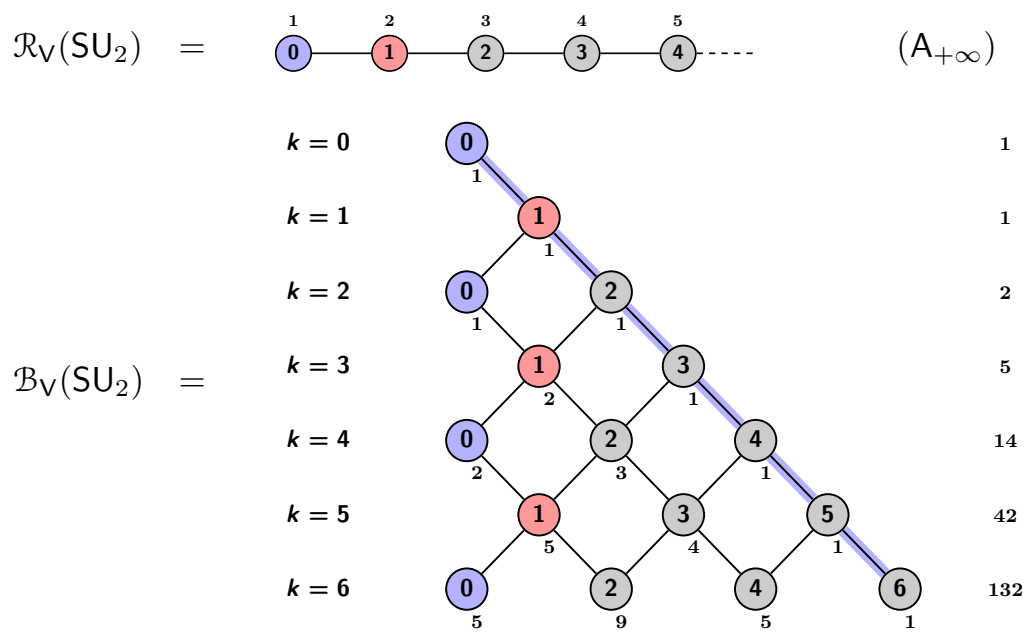

Fig. 1: The representation graph $\mathcal{R}_{V}\left(\mathrm{SU}_{2}\right)$, which is the Dynkin diagram $\mathrm{A}_{+\infty}$, and the first 6 levels of the corresponding Bratteli diagram $\mathcal{B}_{\vee}\left(\mathrm{SU}_{2}\right)$. In $\mathcal{R}_{\mathrm{V}}\left(\mathrm{SU}_{2}\right)$, the label on the node is the index of the $\mathrm{SU}_{2}$-module, and the label above the node is its dimension. The trivial module is shown in blue and the defining module $\vee$ in red. In $\mathcal{B}_{\vee}\left(S_{2}\right)$ the label below vertex $r$ on level $k$ gives the number of paths (Dyck paths in this case) from the top of the diagram to $r$, which is multiplicity of $\mathrm{V}(r)$ in $\mathrm{V}^{\otimes k}$. These numbers also count the walks of length $k$ from 0 to $r$ on $\mathcal{R}_{\mathrm{V}}(\mathrm{G})$. The column to the right contains the sum of the squares of the multiplicities, which at level $k$ is the Catalan number $\mathcal{C}_{k}$ and equals the dimension of the centralizer algebra $\mathrm{Z}_{k}\left(\mathrm{SU}_{2}\right)$.

occur in some $\mathrm{V}^{\otimes k}$, and thus $\mathcal{R}_{\mathrm{V}}(\mathrm{G})$ is connected. The representation graphs $\mathcal{R}_{\mathrm{V}}(\mathrm{G})$ corresponding to $\mathbf{G}=\mathbf{C}_{n}, \mathbf{D}_{n}, \mathbf{T}, \mathbf{O}, \mathbf{I}$, are displayed in Figure 2. McKay observed that these graphs correspond exactly to the affine Dynkin diagrams of type $\hat{A}_{n-1}, \hat{D}_{n+2}, \hat{E}_{6} \hat{E}_{7}, \hat{E}_{8}$, respectively. The trivial module $G^{(0)}$ corresponds to the affine node in those cases.

For $k \geq 1$, the $k$-fold tensor power $\mathrm{V}^{\otimes k}$ is $2^{k}$-dimensional and has a basis of simple tensors $\mathrm{V} \otimes k=$ $\operatorname{span}_{\mathbb{C}}\left\{\mathbf{v}_{r_{1}} \otimes \mathrm{v}_{r_{2}} \otimes \cdots \otimes \mathrm{v}_{r_{k}} \mid r_{j} \in\{-1,1\}\right\}$. Group elements $g \in \mathrm{G}$ act on simple tensors by the diagonal action $g\left(\mathrm{v}_{r_{1}} \otimes \mathrm{v}_{r_{2}} \otimes \cdots \otimes \mathrm{v}_{r_{k}}\right)=g \mathrm{v}_{r_{1}} \otimes g \mathrm{v}_{r_{2}} \otimes \cdots \otimes g \mathrm{v}_{r_{k}}$. Let

$$
\Lambda_{k}(\mathrm{G})=\left\{\lambda \in \Lambda(\mathrm{G}) \mid \mathrm{G}^{\lambda} \text { appears as a summand in the decomposition of } \mathrm{V}^{\otimes k}\right\}
$$

Then, $\Lambda_{k}(\mathrm{G})$ is the set of vertices in $\mathcal{R}_{\mathrm{V}}(\mathrm{G})$ that can be reached by paths of length $k$ starting from 0 . Furthermore, $\Lambda_{k}(\mathrm{G}) \subseteq \Lambda_{k+2}(\mathrm{G})$, for all $k \geq 0$, since if a node can be reached in $k$ steps, then it can also be reached in $k+2$ steps. The Bratteli diagram $\mathcal{B}_{\mathrm{V}}(\mathrm{G})$ is the infinite graph with vertices labeled by $\Lambda_{k}(\mathrm{G})$ on level $k$ and $a_{\lambda, \mu}$ edges from vertex $\lambda \in \Lambda_{k}(\mathrm{G})$ to vertex $\mu \in \Lambda_{k+1}(\mathrm{G})$. The Bratteli diagram for $\mathbf{G}=\mathrm{SU}_{2}$ is shown in Figure 1, and the Bratteli diagrams for $\mathbf{G}=\mathbf{C}_{n}, \mathbf{D}_{n}, \mathbf{T}, \mathbf{O}, \mathbf{I}$ are shown in Figure 3 .

A walk of length $k$ on the representation graph $\mathcal{R}_{\mathrm{V}}(\mathrm{G})$ from 0 to $\lambda \in \Lambda(\mathrm{G})$, is a sequence $\left(0, \lambda^{1}, \lambda^{2}, \ldots\right.$, $\left.\lambda^{k}=\lambda\right)$ starting at $\lambda^{0}=0$, such that $\lambda^{j} \in \Lambda(\mathrm{G})$ for each $1 \leq j \leq k$, and $\lambda^{j-1}$ is connected to $\lambda^{j}$ by an edge in $\mathcal{R}_{\mathrm{V}}(\mathrm{G})$. Such a walk is equivalent to a unique path of length $k$ on the Bratteli diagram $\mathcal{B}_{\mathrm{V}}(\mathrm{G})$ from $0 \in \Lambda_{0}(\mathrm{G})$ to $\lambda \in \Lambda_{k}(\mathrm{G})$. Let $\mathcal{W}_{k}^{\lambda}(\mathrm{G})$ denote the set of walks on $\mathcal{R}_{\mathrm{V}}(\mathrm{G})$ of length $k$ from $0 \in \Lambda(\mathrm{G})$ 


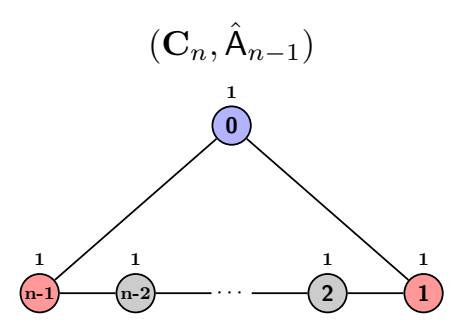

$\left(\mathbf{T}, \hat{\mathrm{E}}_{6}\right)$

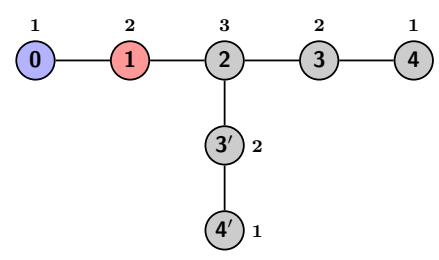

$\left(\mathbf{D}_{n}, \hat{\mathrm{D}}_{n+2}\right)$

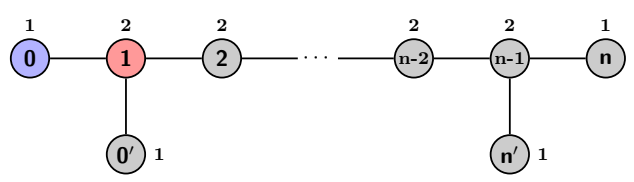

$\left(\mathbf{O}, \hat{E}_{7}\right)$

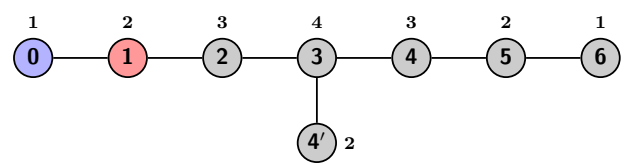

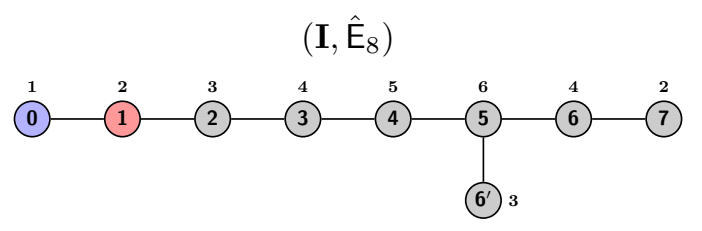

Fig. 2: The representation graphs $\mathcal{R}_{\mathrm{V}}(\mathrm{G})$ for the finite subgroups $\mathrm{G}=\mathbf{C}_{n}, \mathbf{D}_{n}, \mathbf{T}, \mathbf{O}, \mathbf{I}$ correspond to the affine Dynkin diagrams of type $\hat{\mathrm{A}}_{n-1}, \hat{\mathrm{D}}_{n+1}, \hat{\mathrm{E}}_{6}, \hat{\mathrm{E}}_{7}, \hat{\mathrm{E}}_{8}$. The label on the node is the index of the G-module, and the label above the node is its dimension. The trivial module (affine node) is blue and the defining module $\mathrm{V}$ is red.

to $\lambda \in \Lambda(\mathrm{G})$, and let $\mathcal{P}_{k}^{\lambda}(\mathrm{G})$ denote the set of paths on $\mathcal{B}_{\mathrm{V}}(\mathrm{G})$ of length $k$ from $0 \in \Lambda_{0}(\mathrm{G})$ to $\lambda \in \Lambda_{k}(\mathrm{G})$. Let $m_{k}^{\lambda}$ denote the multiplicity of $\mathrm{G}^{\lambda}$ in $\mathrm{V}^{\otimes k}$. Then, by induction on (2) we have

$$
\begin{aligned}
m_{k}^{\lambda}=\left|\mathcal{W}_{k}^{\lambda}(\mathrm{G})\right|=\#\left(\text { walks on } \mathcal{R}_{\mathrm{V}}(\mathrm{G}) \text { of length } k \text { from at } 0 \text { to } \lambda\right) \\
=\left|\mathcal{P}_{k}^{\lambda}(\mathrm{G})\right|=\#\left(\text { paths in } \mathcal{B}_{\mathrm{V}}(\mathrm{G}) \text { of length } k \text { from } 0 \in \Lambda_{0}(\mathrm{G}) \text { to } \lambda \in \Lambda_{k}(\mathrm{G})\right) .
\end{aligned}
$$

The centralizer of $\mathrm{G}$ on $\mathrm{V} \otimes k$ is the algebra

$$
\mathrm{Z}_{k}(\mathrm{G})=\operatorname{End}_{\mathrm{G}}\left(\mathrm{V}^{\otimes k}\right)=\left\{a \in \operatorname{End}\left(\mathrm{V}^{\otimes k}\right) \mid a(g w)=g a(w) \text { for all } g \in \mathrm{G}, w \in \mathrm{V}^{\otimes k}\right\} .
$$

If the group $G$ is apparent from the context, we will simply write $Z_{k}$ for $Z_{k}(G)$. Since $V^{\otimes 0}=G^{(0)}$, we have $\mathrm{Z}_{0}(\mathrm{G})=\mathbb{C} 1$. There is a natural embedding $\mathrm{Z}_{k}(\mathrm{G}) \hookrightarrow \mathrm{Z}_{k+1}(\mathrm{G})$ given by $a \mapsto a \otimes \mathbf{1}$, where $a \otimes \mathbf{1}$ acts as $a$ on the first $k$ tensor factors and $\mathbf{1}$ acts as the identity in the $(k+1)$ st tensor position. Iterating this embedding gives an infinite tower of algebras $\mathrm{Z}_{0}(\mathrm{G}) \subseteq \mathrm{Z}_{1}(\mathrm{G}) \subseteq \mathrm{Z}_{2}(\mathrm{G}) \subseteq \cdots$.

By classical double-centralizer theory (see for example [6, 3B,68]), we have:

- $\mathrm{Z}_{k}(\mathrm{G})$ is a semisimple $\mathbb{C}$-algebra with irreducible modules $\left\{\mathrm{Z}_{k}^{\lambda} \mid \lambda \in \Lambda_{k}(\mathrm{G})\right\}$ labeled by $\Lambda_{k}(\mathrm{G})$. 
- $\operatorname{dim} Z_{k}^{\lambda}=m_{k}^{\lambda}=\left|\mathcal{W}_{k}^{\lambda}(\mathrm{G})\right|=\left|\mathcal{P}_{k}^{\lambda}(\mathrm{G})\right|$.

- Edges from level $k$ to $k-1$ in $\mathcal{B}_{\vee}(\mathrm{G})$ represent restriction and induction rules for $\mathrm{Z}_{k-1}(\mathrm{G}) \subseteq \mathrm{Z}_{k}(\mathrm{G})$.

- If $d^{\lambda}=\operatorname{dim} \mathrm{G}^{\lambda}$, then the tensor space $\mathrm{V}^{\otimes k}$ has the following decomposition

$$
\mathrm{V}^{\otimes k} \cong \underbrace{\bigoplus_{\lambda \in \Lambda_{k}(\mathrm{G})} m_{k}^{\lambda} \mathrm{G}^{\lambda}}_{\text {as a G-module }} \cong \underbrace{\bigoplus_{\lambda \in \Lambda_{k}(\mathrm{G})} d^{\lambda} \mathrm{Z}_{k}^{\lambda}}_{\text {as a } \mathrm{Z}_{k}(\mathrm{G}) \text {-module }} \cong \underbrace{\bigoplus_{\lambda \in \Lambda_{k}(\mathrm{G})}\left(\mathrm{G}^{\lambda} \otimes \mathrm{Z}_{k}^{\lambda}\right)}_{\text {as a }\left(\mathrm{G}, \mathrm{Z}_{k}(\mathrm{G})\right) \text {-bimodule }} .
$$

- By general Wedderburn theory, the dimension of $Z_{k}(G)$ is

$$
\operatorname{dim} Z_{k}(\mathrm{G})=\sum_{\lambda \in \Lambda_{k}(\mathrm{G})}\left(m_{k}^{\lambda}\right)^{2}=\sum_{\lambda \in \Lambda_{k}(\mathrm{G})}\left|\mathcal{W}_{k}^{\lambda}(\mathrm{G})\right|^{2}=\left|\mathcal{W}_{2 k}^{0}(\mathrm{G})\right|=\operatorname{dim} \mathrm{Z}_{2 k}^{(0)}
$$

which equals the number of walks of length $2 k$ that begin and end at 0 on $\mathcal{R}_{\mathrm{V}}(\mathrm{G})$. The third equality follows from the property that a pair of walks of length $k$ from 0 to $\lambda$ corresponds uniquely (by reversing the second walk) to a walk of length $2 k$ beginning and ending at 0 .

\section{Paths and Dimensions}

Using an inductive argument on the structure of the Bratteli diagram, we compute the dimensions of the irreducible $Z_{k}(G)$-modules $Z_{k}^{\lambda}$ for $\lambda \in \Lambda_{k}(G)$. They are given explicitly in [1]. This dimension also equals the multiplicity of $\mathrm{G}^{\lambda}$ in $\mathrm{V}^{\otimes k}$. The dimension of the centralizer algebra $\mathrm{Z}_{k}(\mathrm{G})$ is then the sum of the squares of these dimensions: $\operatorname{dim} Z_{k}(G)=\sum_{\lambda} \operatorname{dim}\left(Z_{k}^{\lambda}\right)^{2}$.

Theorem 8 ([1] Dimension Formulas) For $k \geq 1$, the following formulas give the dimension $\operatorname{dim} \mathrm{Z}_{k}(\mathrm{G})$ of the McKay centralizer algebra, which also equals the number of $2 k$-walks on the representation graph $\mathcal{R}_{\mathrm{V}}(\mathrm{G})$ from 0 to 0 .

(a) $\operatorname{dim} Z_{k}\left(\mathbf{C}_{n}\right)=2 \operatorname{dim} Z_{k}\left(\mathbf{D}_{n}\right)=\sum_{\substack{0 \leq a, b \leq k \\ a \equiv b \bmod \tilde{n}}}\left(\begin{array}{l}k \\ a\end{array}\right)\left(\begin{array}{l}k \\ b\end{array}\right)=$ coefficient of $z^{k}$ in $\left.(1+z)^{2 k}\right|_{z^{\tilde{n}}=1}$ which equals the the $2 k-k$ coefficient in Pascal's triangle on a cylinder of "diameter" $\tilde{n}$ (Fig. 3), where $\tilde{n}=n$, if $n$ is odd, and $\tilde{n}=\frac{1}{2} n$, if $n$ is even.

(b) $\operatorname{dim} Z_{k}(\mathbf{T})=\frac{4^{k}+8}{12}([14]$ OEIS sequence A047849).

(c) $\operatorname{dim} Z_{k}(\mathbf{O})=\frac{4^{k}+6 \cdot 2^{k}+8}{24}([14]$ OEIS sequence A007581).

(d) $\operatorname{dim} Z_{k}(\mathbf{I})=\frac{4^{k}+12 L_{2 k}+20}{60}$, where $L_{n}$ is the Lucas number defined by $L_{0}=2, L_{1}=1$, and $L_{n+2}=L_{n+1}+L_{n}$. 
$\mathbf{C}_{5}$

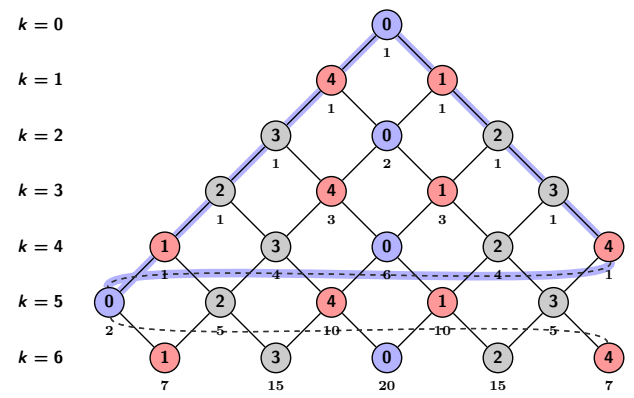

$\mathbf{D}_{6}:$

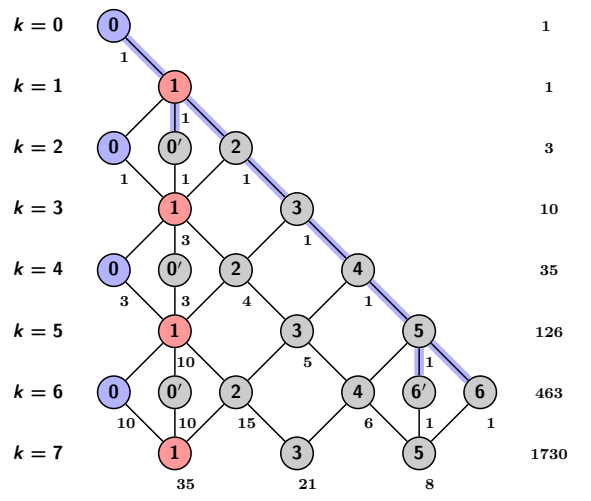

O :

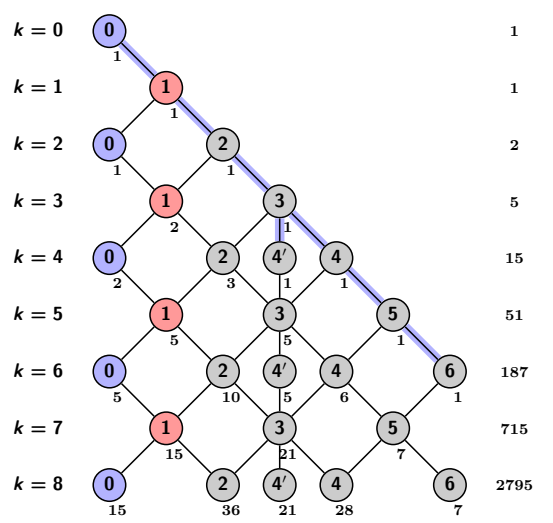

$$
\mathbf{C}_{10} \text { : }
$$

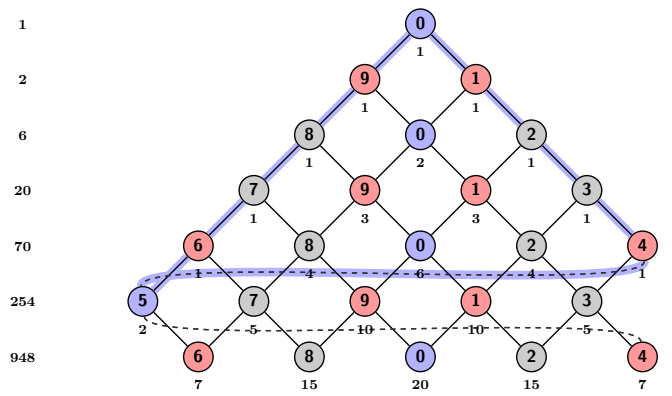

T :

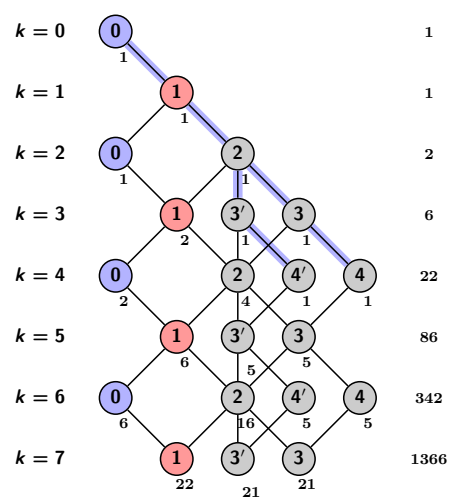

I :

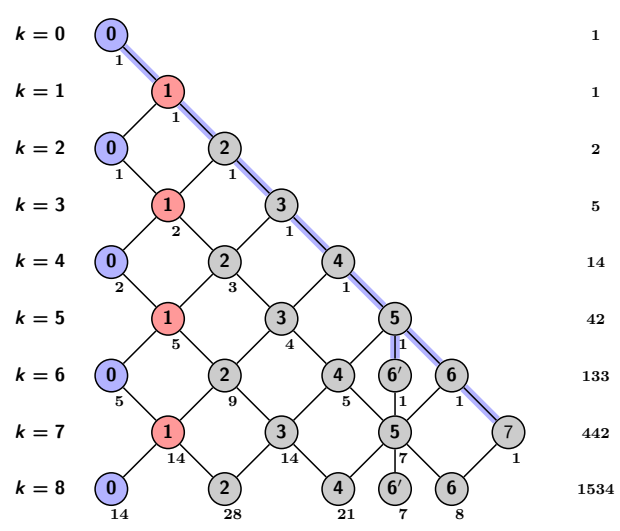

Fig. 3: The first several rows of the Bratteli diagrams $\mathcal{B}_{V}(G)$ for $G=\mathbf{C}_{5}, \mathbf{C}_{10}, \mathbf{D}_{6}, \mathbf{T}, \mathbf{O}, \mathbf{I}$. The representation graph $\mathcal{R}_{V}(\mathrm{G})$ is embedded as the shaded edges. The unshaded edges are reflections from the row above and correspond to the Jones basic construction ideal $Z_{k} e_{k} Z_{k}$ (see Sec. 4). The Bratteli diagrams for $\mathbf{C}_{5}$ and $\mathbf{C}_{10}$ are isomorphic. The label below vertex $r$ on level $k$ gives the number of $k$-paths from the top of the diagram to $r$, which is also multiplicity of $\mathrm{G}^{(r)}$ in $\mathrm{V}^{\otimes k}$. These numbers also give the number of $k$-walks from 0 to $r$ on the representation graph $\mathcal{R}_{\mathrm{V}}(\mathrm{G})$. The column to the right contains the sum of the squares of the multiplicities which equals $\operatorname{dim} Z_{k}(G)$. 


\section{Basic Construction}

In this section we define two kinds of (essential) idempotents $\left\{\mathrm{e}_{i} \mid 1 \leq i \leq k-1\right\}$ and $\left\{\mathrm{f}_{\nu} \mid \nu \in \Lambda(\mathrm{G})\right\}$ which together with 1 generate $Z_{k}(\mathrm{G})$. For $1 \leq i \leq k-1$, define an endomorphism $\mathrm{e}_{i}$ on $\mathrm{V}^{\otimes k}$ by

$$
\mathrm{e}_{i}=\mathbf{1} \otimes \cdots \otimes \mathbf{1} \otimes \mathrm{e} \otimes \mathbf{1} \otimes \cdots \otimes \mathbf{1},
$$

where 1 is the $2 \times 2$ identity matrix, and $\mathrm{e}: \mathrm{V} \otimes \mathrm{V} \rightarrow \mathrm{V} \otimes \mathrm{V}$ acts in tensor positions $i$ and $i+1$ by $\mathrm{e}\left(\mathrm{v}_{j} \otimes \mathrm{v}_{\ell}\right)=\mathrm{v}_{j} \otimes \mathrm{v}_{\ell}-\mathrm{v}_{\ell} \otimes \mathrm{v}_{j}$, for $j, \ell \in\{-1,1\}$. Thus, $\mathrm{e}: \mathrm{V}^{\otimes 2} \rightarrow \mathrm{V}^{\otimes 2}$ projects onto the antisymmetric tensors in $\mathrm{V}^{\otimes 2}$, and it is easy to confirm that $\mathrm{e}_{i} \in \mathrm{Z}_{k}(\mathrm{G})$.

For $\nu \in \Lambda(\mathrm{G})$, we let $|\nu|$ equal the distance from 0 to $\nu$ in the representation graph $\mathcal{R}_{\mathrm{V}}(\mathrm{G})$. Thus by the way we have chosen our labels in Figure $3 .|(\ell)|=\left|\left(\ell^{\prime}\right)\right|=\ell$. Correspondingly, the module $\mathrm{G}^{\nu}$ first appears as a constituent of $\mathrm{V} \otimes k$, when $k=|\nu|$, and it appears in that tensor product with multiplicity exactly 1 . Define

$$
\mathrm{f}_{\nu}:=\text { the } \mathrm{G} \text {-module homomorphism projecting onto the unique copy of } \mathrm{G}^{\nu} \text { in } \mathrm{V}^{\otimes|\nu|} \text {. }
$$

In [1] we show how to explicitly construct $\mathrm{f}_{\nu}$ for each $\nu \in \Lambda(\mathrm{G})$. In particular, we show that

$$
\mathrm{f}_{\nu}-\frac{d^{\nu-1}}{d^{\nu}} \mathrm{f}_{\nu} \mathrm{e}_{|\nu|} \mathrm{f}_{\nu}=\sum_{\mu=\nu+1} \mathrm{f}_{\mu}
$$

where $\nu-1 \in \Lambda(\mathrm{G})$ is the unique neighbor of $\nu$ in $\mathcal{R}_{\mathrm{V}}(\mathrm{G})$ that is closer to 0 (i.e., $|\nu-1|=|\nu|-1$ ), where the sum is over the neighbors $\mu=\nu+1$ of $\nu$ in $\mathcal{R}_{\mathrm{V}}(\mathrm{G})$ that are farther from 0 (i.e., $|\mu|=|\nu|+1$ ), and where $d^{\lambda}=\operatorname{dim} \mathrm{G}^{\lambda}$. In most cases, $\nu$ has degree 2 , and there is a unique node of the form $\mu=$ $\nu+1$, and thus (10) defines $\mathrm{f}_{\mu}$ uniquely. If $\nu$ has degree greater than 2 , then we use other methods [1, Sec. 1.8] to decompose the sum into the constituent $\mathrm{f}_{\mu}$. This recursive construction is a generalization of the construction of the Jones-Wenzl idempotent for $\mathrm{SU}_{2}$.

A branch node in the representation graph $\mathcal{R}_{\mathrm{V}}(\mathrm{G})$ is any vertex of degree greater than 2. Let $\mathrm{br}(\mathrm{G})$ denote the set of branch nodes in $\mathcal{R}_{\mathrm{V}}(\mathrm{G})$. In the special case of $\mathcal{R}_{\mathrm{V}}\left(\mathbf{C}_{n}\right)$ for $n \leq \infty$, we consider the affine node to be the branch node. Let the diameter of $\mathcal{R}_{\mathrm{V}}(\mathrm{G})$, denoted by $\operatorname{diam}(\mathrm{G})$, be the maximum distance between any vertex $\lambda \in \Lambda(\mathrm{G})$ and $0 \in \Lambda(\mathrm{G})$. For $\mathrm{G}=\mathbf{C}_{n}$, we let $\operatorname{diam}(\mathrm{G})=\tilde{n}$ as in 11.

\begin{tabular}{c|cccccccc}
$\mathrm{G}$ & $\mathrm{SU}_{2}$ & $\mathbf{C}_{n}$ & $\mathbf{D}_{n}$ & $\mathbf{T}$ & $\mathbf{O}$ & $\mathbf{I}$ & $\mathbf{C}_{\infty}$ & $\mathbf{D}_{\infty}$ \\
\hline $\operatorname{diam}(\mathrm{G})$ & $\infty$ & $\tilde{n}$ & $n$ & 4 & 6 & 7 & $\infty$ & $\infty$ \\
$\operatorname{br}(\mathrm{G})$ & $\emptyset$ & $\{0\}$ & $\{1, n\}$ & $\{2\}$ & $\{3\}$ & $\{5\}$ & $\{0\}$ & $\{1\}$
\end{tabular}$\quad \tilde{n}=\left\{\begin{array}{cl}\frac{1}{2} n, & \text { if } n \text { is even, } \\
n, & \text { if } n \text { is odd. }\end{array}\right.$

The Jones basic construction (see [8], [16], [9]) uses the ideal $\mathrm{Z}_{k} \mathrm{e}_{k} \mathrm{Z}_{k} \subseteq \mathrm{Z}_{k+1}$ to recursively study the structure of $Z_{k+1}$. We use it to prove the following theorem.

Theorem 12 ([1] Basic Construction) Let $\mathrm{Z}_{k}=\mathrm{Z}_{k}(\mathrm{G})$ and let $\mathrm{Z}_{0}=\mathbb{C} 1$. Then for $k \geq 1$, except for the two special cases in part (d), we have

(a) $Z_{k+1}=Z_{k} e_{k} Z_{k} \oplus N_{k+1}$, where $Z_{k} e_{k} Z_{k}$ is a two-sided basic construction ideal, and $N_{k+1}$ is a commutative subalgebra spanned by $\left\{\mathrm{f}_{\nu}|| \nu \mid=k+1\right\}$. In particular, $\mathrm{N}_{k+1}=0$ if $k \geq \operatorname{diam}(\mathrm{G})$.

(b) $\mathrm{Z}_{k+1}=\left\langle\mathrm{Z}_{k}, \mathrm{e}_{k}\right\rangle$ if $k \notin \mathrm{br}(\mathrm{G})$, and $\mathrm{Z}_{k+1}=\left\langle\mathrm{Z}_{k}, \mathrm{e}_{k}, \mathrm{f}_{(k+1)}\right\rangle$ if $k \in \mathrm{br}(\mathrm{G})$. 
(c) $\mathrm{Z}_{k+1}$ is generated by $\left\{1, \mathrm{e}_{1}, \ldots, \mathrm{e}_{k}\right\} \cup\left\{\mathrm{f}_{(\ell+1)} \mid \ell \in \operatorname{br}(\mathrm{G}), \ell \leq k\right\}$.

(d) $\mathrm{TL}_{k}(2) \subseteq \mathrm{Z}_{k}$ for all $k \geq 0$, and $\mathrm{TL}_{k}(2)=\mathrm{Z}_{k}$ for $0 \leq k \leq \min (\operatorname{br}(\mathrm{G}))$.

(e) Two special cases: (i) if $\mathrm{G}=\mathbf{C}_{n}$, then $\mathrm{Z}_{\tilde{n}}=\left\langle\mathrm{Z}_{\tilde{n}-1}, \mathrm{e}_{\tilde{n}-1}, \mathrm{E}_{\mathrm{p}, \mathrm{q}}\right.$, for $\left.\mathrm{p}, \mathrm{q} \in\{-\underline{1}, \underline{1}\}\right\rangle$, where $\mathrm{E}_{\mathrm{p}, \mathrm{q}}$ are matrix units. (ii) if $\mathrm{G}=\mathbf{D}_{2}$, then $\mathrm{Z}_{2}=\left\langle\mathrm{Z}_{1}, \mathrm{e}_{1}, \mathrm{f}_{\mu_{1}}, \mathrm{f}_{\mu_{2}}\right\rangle$ where $\mu_{1}, \mu_{2} \in\left\{\left(0^{\prime}\right),(2),\left(2^{\prime}\right)\right\}, \mu_{1} \neq \mu_{2}$.

Example 13 Compare these examples with their representation graphs $\mathcal{R}_{\mathrm{V}}(\mathrm{G})$ in Figure 2 .

(a) If $\mathrm{G}=\mathbf{O}$, then $\operatorname{br}(\mathbf{O})=\{3\}, \operatorname{diam}(\mathbf{O})=6$, and

$$
\begin{array}{ll}
\mathrm{Z}_{1}=\mathbb{C} 1=\mathbb{C f}\left(\mathrm{f}_{(1)}=\mathrm{Z}_{0} \cong \mathrm{TL}_{1}(2)\right. & \mathrm{Z}_{5}=\mathrm{Z}_{4} \mathrm{e}_{4} \mathrm{Z}_{4} \oplus \mathbb{C f}(5)=\left\langle\mathrm{Z}_{4}, \mathrm{e}_{4}\right\rangle \\
\mathrm{Z}_{2}=\mathrm{Z}_{1} \mathrm{e}_{1} \mathrm{Z}_{1} \oplus \mathbb{C f}(2)=\left\langle\mathrm{Z}_{1}, \mathrm{e}_{1}\right\rangle \cong \mathrm{TL}_{2}(2) & \mathrm{Z}_{6}=\mathrm{Z}_{5} \mathrm{e}_{5} \mathrm{Z}_{5} \oplus \mathbb{C f}(6)=\left\langle\mathrm{Z}_{5}, \mathrm{e}_{5}\right\rangle \\
\mathrm{Z}_{3}=\mathrm{Z}_{2} \mathrm{e}_{2} \mathrm{Z}_{2} \oplus \mathbb{C f}\left(\mathrm{Cf}_{(3)}=\left\langle\mathrm{Z}_{2}, \mathrm{e}_{2}\right\rangle \cong \mathrm{TL}_{3}(2)\right. & \mathrm{Z}_{7}=\mathrm{Z}_{6} \mathrm{e}_{6} \mathrm{Z}_{6}=\left\langle\mathrm{Z}_{6}, \mathrm{e}_{6}\right\rangle \\
\mathrm{Z}_{4}=\mathrm{Z}_{3} \mathrm{e}_{3} \mathrm{Z}_{3} \oplus \mathbb{C f} \mathrm{Cf}_{(4)} \oplus \mathbb{C f}\left(\mathrm{Cf}^{\prime}\right)=\left\langle\mathrm{Z}_{3}, \mathrm{e}_{3}, \mathrm{f}_{(4)}\right\rangle & \mathrm{Z}_{k+1}=\mathrm{Z}_{k} \mathrm{e}_{k} \mathrm{Z}_{k}=\left\langle\mathrm{Z}_{k}, \mathrm{e}_{k}\right\rangle, k \geq 6 .
\end{array}
$$

(b) If $\mathrm{G}=\mathbf{D}_{6}$, then $\operatorname{br}\left(\mathbf{D}_{6}\right)=\{1,5\}$, $\operatorname{diam}\left(\mathbf{D}_{6}\right)=6$, and

$$
\begin{array}{ll}
\mathrm{Z}_{1}=\mathbb{C} 1=\mathbb{C f}_{(1)}=\mathrm{Z}_{0} \cong \mathrm{TL}_{1}(2) & \mathrm{Z}_{5}=\mathrm{Z}_{4} \mathrm{e}_{4} \mathrm{Z}_{4} \oplus \mathbb{C f} f_{(5)}=\left\langle\mathrm{Z}_{4}, \mathrm{e}_{4}\right\rangle \\
\mathrm{Z}_{2}=\mathrm{Z}_{1} \mathrm{e}_{1} \mathrm{Z}_{1} \oplus \mathbb{C f}\left(0 _ { ( 0 ^ { \prime } ) } \oplus \mathbb { C f } \left(\mathrm{Cf}_{(2)}=\left\langle\mathrm{Z}_{1}, \mathrm{e}_{1}, \mathrm{f}_{(2)}\right\rangle\right.\right. & \mathrm{Z}_{6}=\mathrm{Z}_{5} \mathrm{e}_{5} \mathrm{Z}_{5} \oplus \mathbb{C f}\left(6_{6}^{\prime}\right) \oplus \mathbb{C f}(6)=\left\langle\mathrm{Z}_{5}, \mathrm{e}_{5}, \mathrm{f}_{(6)}\right\rangle \\
\mathrm{Z}_{3}=\mathrm{Z}_{2} \mathrm{e}_{2} \mathrm{Z}_{2} \oplus \mathbb{C f}\left(\mathrm{f}_{(3)}=\left\langle\mathrm{Z}_{2}, \mathrm{e}_{2}\right\rangle\right. & \mathrm{Z}_{7}=\mathrm{Z}_{6} \mathrm{e}_{6} \mathrm{Z}_{6}=\left\langle\mathrm{Z}_{6}, \mathrm{e}_{6}\right\rangle \\
\mathrm{Z}_{4}=\mathrm{Z}_{3} \mathrm{e}_{3} \mathrm{Z}_{3} \oplus \mathbb{C f} f_{(4)}=\left\langle\mathrm{Z}_{3}, \mathrm{e}_{3}\right\rangle & \mathrm{Z}_{k+1}=\mathrm{Z}_{k} \mathrm{e}_{k} \mathrm{Z}_{k}=\left\langle\mathrm{Z}_{k}, \mathrm{e}_{k}\right\rangle, k \geq 6 .
\end{array}
$$

\section{Linear Bases}

Let $\mathcal{P}_{2 k}^{0}(\mathrm{G})$ denote the set of paths on $\mathcal{B}_{\mathrm{V}}(\mathrm{G})$ of length $2 k$ from 0 at level 0 to 0 at level $2 k$. Then $\operatorname{dim} Z_{k}(\mathrm{G})=\left|\mathcal{P}_{2 k}^{0}(\mathrm{G})\right|$, and so it is natural to seek a basis $\left\{w_{p} \mid p \in \mathcal{P}_{2 k}^{0}(\mathrm{G})\right\}$ of $\mathrm{Z}_{k}(\mathrm{G})$ where each $w_{p}$ is a word in the generators $\left\{1, \mathrm{e}_{1}, \ldots, \mathrm{e}_{k-1}\right\} \cup\left\{\mathrm{f}_{(\ell+1)} \mid \ell \in \operatorname{br}(\mathrm{G}), \ell<k\right\}$. For example, when $\mathrm{G}=\mathrm{SU}_{2}$, the centralizer is the Temperley-Lieb algebra $\mathrm{Z}_{k}\left(\mathrm{SU}_{2}\right) \cong \mathrm{TL}_{k}(2)$, the dimension is the Catalan number $\mathcal{C}_{k}$, the paths are Dyck paths, and the bijection to a basis of words in $\mathrm{e}_{1}, \ldots, \mathrm{e}_{k-1}$ is given in [8, 2.8]. Here we generalize this result uniformly to the finite subgroups $\mathrm{G} \subseteq \mathrm{SU}_{2}$.

For $\ell=\min (\operatorname{br}(G))$, i.e., the first branch point, define

$$
\mathrm{b}_{i}=\underbrace{1 \otimes \cdots \otimes 1}_{(i-1) \text { factors }} \otimes \mathrm{f}_{(\ell+1)} \otimes \underbrace{1 \otimes \cdots \otimes 1}_{(k-i-\ell) \text { factors }}, \quad 1 \leq i \leq k-\ell, \quad \text { for each } \mathrm{G},
$$

and define

$$
\mathrm{c}_{i}=\underbrace{\mathbf{1} \otimes \cdots \otimes \mathbf{1}}_{(i-1) \text { factors }} \otimes \mathrm{f}_{(n)} \otimes \underbrace{1 \otimes \cdots \otimes \mathbf{1}}_{(k-i-n+1) \text { factors }}, \quad 1 \leq i \leq k-n+1, \quad \text { for } \mathbf{G}=\mathbf{D}_{n} .
$$

A path $p \in \mathcal{P}_{2 k}^{0}$ is a sequence $p=\left(0=p_{0}, p_{1}, \ldots, p_{2 k-1}, p_{2 k}=0\right)$, where each $p_{i} \in \Lambda_{i}(\mathrm{G})$ is a label of an irreducible G-module that appears in $\mathrm{V}^{\otimes i}$. A peak in a path $p$ is a label $p_{i}$ such that $\left|p_{i-1}\right|<\left|p_{i}\right|$ and $\left|p_{i}\right|>\left|p_{i+1}\right|$. If a peak $p_{i}$ is marked with the prime symbol (e.g., $0_{i}^{\prime}$ or $4_{i}^{\prime}$, etc), then $p_{i}$ is a nonstandard peak. Otherwise it is said to be standard. To each peak $p_{i}$ we associate a product of generators called block as follows

$$
\begin{aligned}
& \mathcal{B}\left(p_{i}\right)=\mathrm{e}_{\alpha} \mathrm{e}_{\alpha-1} \mathrm{e}_{\alpha-2} \cdots \mathrm{e}_{\beta}, \text { with } \alpha=\frac{i+\left|p_{i}\right|-2}{2} \text { and } \beta=\frac{i-\left|p_{i}\right|+2}{2}, \\
& \mathcal{B}\left(p_{i}^{\prime}\right)=\mathrm{b}_{\beta}, \text { with } \beta=\frac{i-\left|p_{i}\right|+2}{2},
\end{aligned}
$$


where the $\mathrm{b}_{\beta}$ is replaced by a $\mathrm{c}_{\beta}$ in $\mathbf{D}_{n}$ if $p_{i}^{\prime}=n_{i}^{\prime}$. When $\mathrm{G}=\mathbf{T}$, we have the following special case,

$$
(\mathrm{G}=\mathbf{T}) \quad \mathcal{B}\left(4_{i}^{\prime}\right)= \begin{cases}\mathrm{b}_{\alpha-2} e_{\alpha} \mathrm{e}_{\alpha-1} \mathrm{e}_{\alpha-2}, \text { with } \alpha=\frac{i+2}{2}, & \text { if } p_{i-2}=2, \\ \mathrm{e}_{\alpha} \mathrm{e}_{\alpha-1} \mathrm{e}_{\alpha-2}, \text { with } \alpha=\frac{i+2}{2}, & \text { if } p_{i-2}=4\end{cases}
$$

If $\mathrm{G}=\mathbf{C}_{n}$ with $n \leq \infty$ then there are further special cases for $\mathcal{B}\left(p_{i}^{\prime}\right)$ and $\mathcal{B}\left(\tilde{n}_{i}^{ \pm}\right)$described in [7].

If $p \in \mathcal{P}_{2 k}^{0}(\mathrm{G})$ is a path in the Bratteli diagram, then we define the word $w_{p}$ of $p$ as the product blocks for each peak in $p$ (see Example 19):

$$
w_{p}=\mathcal{B}\left(p_{i_{1}}\right) \mathcal{B}\left(p_{i_{2}}\right) \cdots \mathcal{B}\left(p_{i_{\ell}}\right), \quad \text { where } p_{i_{1}}, p_{i_{2}}, \ldots, p_{i_{\ell}} \text { are the peaks in } p \text {. }
$$

Theorem 18 (|7| Basis Theorem) For $\mathrm{G}=\mathrm{SU}_{2}, \mathbf{C}_{n}, \mathbf{D}_{n}, \mathbf{C}_{\infty}, \mathbf{D}_{\infty}, \mathbf{T}, \mathbf{O}$, or $\mathbf{I}$, and $k \geq 0$, the set $\left\{w_{p} \mid p \in \mathcal{P}_{2 k}^{0}(\mathrm{G})\right\}$ is a basis for $\mathrm{Z}_{k}(\mathrm{G})$.

Example 19 (Paths and their Corresponding Words) The following are examples of paths $p$ in the Bratteli diagram $\mathcal{B}_{\mathrm{V}}(\mathrm{G})$ with their peaks circled and the corresponding words $w_{p}$.

(a) In $\mathrm{Z}_{10}(\mathbf{O})$

$$
\begin{aligned}
p & =\left(0_{0}, 1_{1}, 2_{2}, 3_{3}, 4_{4}, 5_{5}, 4_{6}, 3_{7}, 4_{8}, 5_{9}, 6_{10}, 5_{11}, 4_{12}, 3_{13}, 2_{14}, 1_{15}, 2_{16}, 3_{17}, 2_{18}, 1_{19}, 0_{20}\right) \\
w_{p} & =\left(\mathrm{e}_{4} \mathrm{e}_{3} \mathrm{e}_{2} \mathrm{e}_{1}\right)\left(\mathrm{e}_{7} \mathrm{e}_{6} \mathrm{e}_{5} \mathrm{e}_{3} \mathrm{e}_{3}\right)\left(\mathrm{e}_{9} \mathrm{e}_{8}\right) \\
p & =\left(0_{0}, 1_{1}, 2_{2}, 3_{3}, 4_{4}, 3_{5}, 4_{6}, 5_{7}, 6_{8}, 5_{9}, 4_{10}, 3_{11}, 4_{12}^{\prime}, 3_{13}, 2_{14}, 1_{15}, 0_{16}, 1_{17}, 2_{18}, 1_{19}, 0_{20}\right) \\
w_{p} & =\left(\mathrm{e}_{3} \mathrm{e}_{2} \mathrm{e}_{1}\right)\left(\mathrm{e}_{6} \mathrm{e}_{5} \mathrm{e}_{4} \mathrm{e}_{3} \mathrm{e}_{2}\right)\left(\mathrm{b}_{3}\right)\left(\mathrm{e}_{9}\right)
\end{aligned}
$$

(b) In $\mathrm{Z}_{10}(\mathbf{T})$

$$
\begin{aligned}
p & =\left(0_{0}, 1_{1}, 2_{2}, 3_{3}, 2_{4}, 3_{5}, 2_{6}, 1_{7}, 0_{8}, 1_{9}, 2_{10}, 3_{11}^{\prime}, 4_{12}^{\prime}, 3_{13}^{\prime}, 2_{14}, 3_{15}, 4_{16}, 3_{17}, 2_{18}, 1_{19}, 0_{20}\right) \\
w_{p} & =\left(\mathrm{e}_{2} \mathrm{e}_{1}\right)\left(\mathrm{e}_{3} \mathrm{e}_{2}\right)\left(\mathrm{b}_{5} \mathrm{e}_{7} \mathrm{e}_{6} \mathrm{e}_{5}\right)\left(\mathrm{e}_{9} \mathrm{e}_{8} \mathrm{e}_{7}\right) \\
p & =\left(0_{0}, 1_{1}, 2_{2}, 3_{3}, 4_{4}, 3_{5}, 4_{6}, 3_{7}, 4_{8}, 3_{9}, 2_{10}, 1_{11}, 0_{12}, 1_{13}, 2_{14}, 3_{15}^{\prime}, 4_{16}^{\prime}, 3_{17}^{\prime}, 2_{18}, 1_{19}, 0_{20}\right) \\
w_{p} & =\left(\mathrm{e}_{3} \mathrm{e}_{2} \mathrm{e}_{1}\right)\left(\mathrm{e}_{4} \mathrm{e}_{3} \mathrm{e}_{2}\right)\left(\mathrm{e}_{5} \mathrm{e}_{4} \mathrm{e}_{3}\right)\left(\mathrm{b}_{7} \mathrm{e}_{9} \mathrm{e}_{8} \mathrm{e}_{7}\right)
\end{aligned}
$$

(c) In $\mathrm{Z}_{10}\left(\mathbf{D}_{5}\right)$

$$
\begin{aligned}
p & =\left(0_{1}, 1_{1}, 2_{2}^{\prime}, 1_{3}, 2_{4}, 3_{5}, 4_{6}, 5_{7}, 4_{8}, 5_{9}^{\prime}, 4_{10}, 3_{11}, 2_{12}, 1_{13}, 0_{14}, 1_{15}, 2_{16}, 1_{17}, 2_{18}^{\prime}, 1_{19}, 0_{20}\right) \\
w_{p} & =\left(\mathrm{b}_{1}\right)\left(\mathrm{e}_{5} \mathrm{e}_{4} \mathrm{e}_{3} \mathrm{e}_{2}\right)\left(\mathrm{c}_{3}\right)\left(\mathrm{e}_{8}\right)\left(\mathrm{b}_{9}\right) \\
p & =\left(0_{0}, 1_{1}, 2_{2}, 3_{3}, 4_{4}, 3_{5}, 4_{6}, 5_{7}, 4_{8}, 3_{9}, 2_{10}, 3_{11}, 4_{12}, 3_{13}, 2_{14}, 1_{15}, 2_{16}, 3_{17}, 2_{18}, 1_{19}, 0_{20}\right) \\
w_{p} & =\left(\mathrm{e}_{3} \mathrm{e}_{2} \mathrm{e}_{1}\right)\left(\mathrm{e}_{5} \mathrm{e}_{4} \mathrm{e}_{3} \mathrm{e}_{2}\right)\left(\mathrm{e}_{7} \mathrm{e}_{6} \mathrm{e}_{5}\right)\left(\mathrm{e}_{9} \mathrm{e}_{8}\right)
\end{aligned}
$$

As above, if $\nu \in \Lambda(\mathrm{G})$, then $|\nu|$ equals the distance from 0 to $\nu$ in $\mathcal{R}_{\mathrm{V}}(\mathrm{G})$. Let $\mathrm{e}_{\nu}:=\mathrm{e}_{|\nu|}$, and let $d^{\nu}=\operatorname{dim} \mathrm{G}^{\nu}$. For $\nu \neq 0, \nu-1 \in \Lambda(\mathrm{G})$ is the unique node that is connected to $\nu$ by an edge with $|\nu-1|=|\nu|-1$. We say that $\mu=\nu+1$ if $\mu \in \Lambda(\mathrm{G})$ is connected to $\nu$ by an edge and $|\mu|=|\nu|+1$. Finally we say that $\mu \prec \nu$ if $\mu$ is on the shortest path from 0 to $\nu$. 
Theorem 20 (Presentation on Generators and Relations) For $k \geq 1$ and $k<\tilde{n}$ if $\mathbf{G}=\mathbf{C}_{n}$, the algebra $\mathrm{Z}_{k}(\mathrm{G})$ is generated by $\left\{1, \mathrm{e}_{1}, \ldots, \mathrm{e}_{k-1}\right\} \cup\left\{\mathrm{f}_{\nu}|\nu \in \Lambda(\mathrm{G}),| \nu \mid \leq k\right\}$ subject to the following relations:

(1) $\mathrm{e}_{i}^{2}=2 \mathrm{e}_{i} ; \quad \mathrm{e}_{i} \mathrm{e}_{i \pm 1} \mathrm{e}_{i}=\mathrm{e}_{i} ; \quad \mathrm{e}_{i} \mathrm{e}_{j}=\mathrm{e}_{j} \mathrm{e}_{i},|i-j|>1$;

(2) $\mathrm{f}_{\nu}^{2}=\mathrm{f}_{\nu} ; \quad \mathrm{f}_{\nu} \mathrm{e}_{i}=\mathrm{e}_{i} \mathrm{f}_{\nu}=0$ for $i<|\nu| ; \quad \mathrm{f}_{\nu} \mathrm{e}_{i}=\mathrm{e}_{i} \mathrm{f}_{\nu}$ for $i>|\nu| ; \quad \mathrm{e}_{\nu} \mathrm{f}_{\nu} \mathrm{e}_{\nu}=\frac{d^{\nu}}{d^{\nu-1}} \mathrm{f}_{\nu-1} \mathrm{e}_{\nu} ;$

(3) $\sum_{\mu=\nu+1} \mathrm{f}_{\mu}=\mathrm{f}_{\nu}-\frac{d^{\nu-1}}{d^{\nu}} \mathrm{f}_{\nu} \mathrm{e}_{\nu-1} \mathrm{f}_{\nu}$ (and this equals 0 if no such $\mu$ exists);

(4) If $|\mu| \leq|\nu|$, then $f_{\mu} f_{\nu}=f_{\nu} f_{\mu}=f_{\mu}$, if $\mu \prec \nu$, and $f_{\mu} f_{\nu}=f_{\nu} f_{\mu}=0$, if $\mu \nprec \nu$.

The case of $k \geq \tilde{n}$, for $\mathrm{G}=\mathbf{C}_{n}$ requires some further notation and is handled in [7].

\section{Exceptional McKay Centralizers and Partition Algebras}

The exceptional groups are referred to as the binary tetrahedral, binary octahedral, and binary icosahedral groups because modulo their centers $Z(G)=\{1,-1\}$, we have the following isomorphisms:

$$
\begin{aligned}
\mathbf{T} /\{1,-1\} \cong \mathbf{A}_{4}, \text { the alternating group on } 4 \text { letters (rotation group of a tetrahedron), } \\
\mathbf{O} /\{1,-1\} \cong \mathbf{S}_{4}, \text { the symmetric group on } 4 \text { letters (rotation group of an octahedron), } \\
\mathbf{I} /\{1,-1\} \cong \mathbf{A}_{5}, \text { the alternating group on } 5 \text { letters (rotation group of an icosahedron). }
\end{aligned}
$$

Group elements act on $\mathrm{V}^{\otimes 2}=\mathrm{V} \otimes \mathrm{V}$ diagonally: $g \cdot\left(\mathrm{v}_{i} \otimes \mathrm{v}_{j}\right)=g \mathrm{v}_{i} \otimes g \mathrm{v}_{j}$. If $g \in \mathcal{Z}(\mathrm{G})=\{1,-1\}$ then $g$ acts on $\vee$ as multiplication by 1 and -1 , so it acts trivially on $\mathrm{V}^{\otimes 2}$. Thus, $Z(\mathrm{G})$ is in the kernel of the action on tensor powers $\mathbf{V}^{\otimes k}$, with $k$ even, and $\mathbf{T}, \mathbf{O}$, and $\mathbf{I}$ act the same as $\mathbf{A}_{4}, \mathbf{S}_{4}$, and $\mathbf{A}_{5}$, respectively

Furthermore, $\operatorname{dim}\left(V^{\otimes 2}\right)=4$, and as a module for $\mathbf{A}_{4}, \mathbf{S}_{4}$, and $\mathbf{A}_{5}$ it decomposes in the following way:

$$
\begin{aligned}
& \mathrm{V}^{\otimes 2} \cong \mathbf{A}_{4}^{(4)} \oplus \mathbf{A}_{4}^{(3,1)} \cong \mathrm{M}, \text { the permutation module for } \mathbf{A}_{4}, \\
& \mathrm{~V}^{\otimes 2} \cong \mathbf{S}_{4}^{(4)} \oplus \mathbf{S}_{4}^{(2,1,1)} \cong \widetilde{\mathrm{M}}, \text { a "twisted" permutation module for } \mathbf{S}_{4}, \\
& \mathrm{~V}^{\otimes 2} \cong \mathbf{A}_{5}^{(5)} \oplus \mathbf{A}_{5}^{(3,1,1)^{+}} \cong \widetilde{\widetilde{M}}, \text { which is not a permutation module for } \mathbf{A}_{5},
\end{aligned}
$$

where here we are using usual integer partition notation to label the irreducible modules for $\mathbf{S}_{n}$ and $\mathbf{A}_{n}$. This means that

$$
\mathrm{Z}_{2 k}(\mathbf{T}) \cong \operatorname{End}_{\mathbf{A}_{4}}\left(\mathrm{M}^{\otimes k}\right), \quad \mathrm{Z}_{2 k}(\mathbf{O}) \cong \operatorname{End}_{\mathbf{S}_{4}}\left(\tilde{\mathrm{M}}^{\otimes k}\right), \quad \mathrm{Z}_{2 k}(\mathbf{I}) \cong \operatorname{End}_{\mathbf{A}_{5}}\left(\widetilde{\widetilde{M}}^{\otimes k}\right) .
$$

The Martin-Jones partition algebras $\mathrm{P}_{k}(n)$ maps surjectively onto $\operatorname{End}_{\mathbf{S}_{n}}\left(\mathrm{M}^{\otimes k}\right)$ for all $n$ and isomorphically for $n \geq 2 k$, where $\mathrm{M}$ is the $n$-dimensional permutation representation of $\mathbf{S}_{n}$ (see [11], [12], [10]). When $\mathrm{M}$ is restricted from $\mathbf{S}_{n}$ to $\mathbf{A}_{n}$ the corresponding partition algebra $\tilde{\mathrm{P}}_{k}(n)$ is studied by Bloss [5]. The partition algebra $\mathrm{P}_{k}(n)$ has a basis indexed by set partitions of $\{1,2, \ldots, 2 k\}$ and a multiplication given by set partition diagram concatenation. See [9] for a survey on partition algebras.

In [3, 4] we study the partition algebras $\operatorname{End}_{\mathbf{S}_{n}}\left(\mathrm{M}^{\otimes k}\right)$ and $\operatorname{End}_{\mathbf{A}_{n}}\left(\mathrm{M}^{\otimes k}\right)$ for $n<2 k$ ("low rank") and for both the permutation module $\mathbf{M}=\mathbf{S}_{n}^{(n)} \oplus \mathbf{S}_{n}^{(n-1,1)}$ and its twisted counterpart $\widetilde{M}=\mathbf{S}_{n}^{(n)} \oplus \mathbf{S}_{n}^{\left(2,1^{n-1}\right)}$. This work gives us alternative precise descriptions of the centralizer algebras $Z_{2 k}(\mathbf{T})$ and $Z_{2 k}(\mathbf{O})$. In [3] we do the following: 


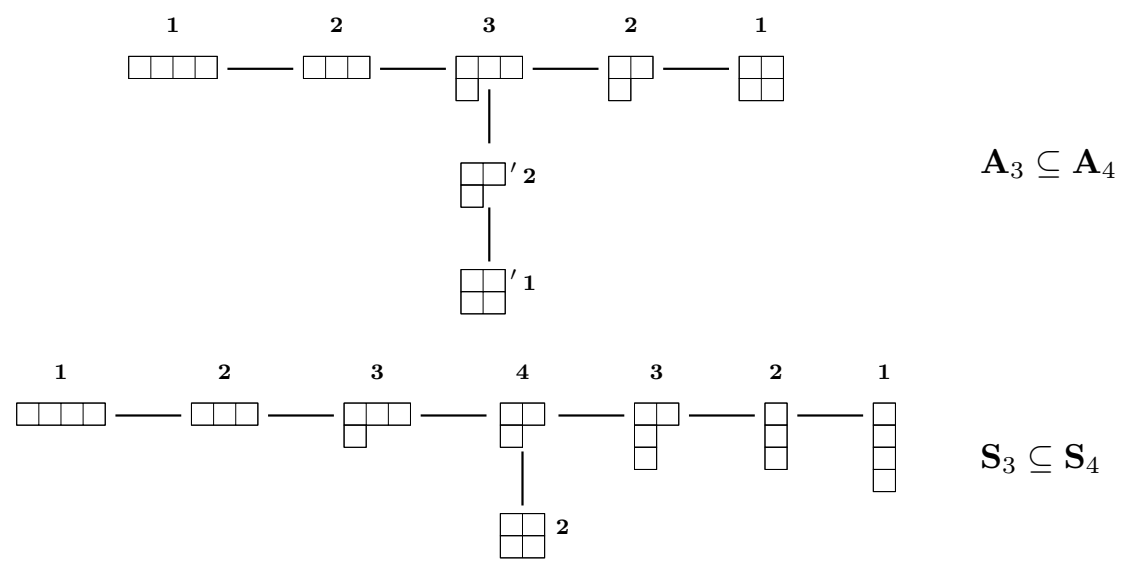

Fig. 4: Restriction-induction graphs for $\mathbf{A}_{3} \subseteq \mathbf{A}_{4}$ and $\mathbf{S}_{3} \subseteq \mathbf{S}_{4}$. These graphs are isomorphic to the representation graphs $\mathcal{R}_{V}(\mathbf{T})$ and $\mathcal{R}_{V}(\mathbf{O})$, respectively, which in turn equal the Dynkin diagrams of type $\hat{\mathrm{E}}_{6}$ and $\hat{\mathrm{E}}_{7}$. See Figure 2

- Explicitly describe the kernel of $\mathrm{P}_{k}(n) \rightarrow \operatorname{End}_{\mathrm{G}}\left(\mathrm{M}^{\otimes k}\right)$, when $n<2 k$, for $\mathrm{G}=\mathbf{S}_{n}, \mathbf{A}_{n}$ and for either the permutation module $\mathbf{M}=\mathbf{S}^{(n)} \oplus \mathbf{S}^{(n-1,1)}$ or its twist $\mathbf{S}^{(n)} \oplus \mathbf{S}^{\left(2,1^{n-1}\right)}$.

- Give two linear bases for the image $\operatorname{End}_{\mathrm{G}}\left(\mathrm{M}^{\otimes k}\right)$ in terms of a restricted collection of set partitions. Combinatorially describe multiplication in both bases and give the change of basis matrix between them in terms of the refinement ordering in the partition lattice $\Pi_{n}$.

Furthermore, these partition algebras can be realized in terms of restriction and induction (this perspective is emphasized in [9]) in the following way. If $\mathbf{U}$ is any $\mathbf{S}_{n}$ module, then upon restriction from $\mathbf{S}_{n}$ to $\mathbf{S}_{n-1}$ followed by induction back to $\mathbf{S}_{n}$ we get $\operatorname{Ind}_{\mathbf{S}_{n-1}} \mathbf{S}_{n} \operatorname{Res}_{\mathbf{S}_{n-1}} \mathbf{S}_{n}(\mathrm{U}) \cong \mathbf{U} \otimes \mathbf{M}$. This is an application of the "tensor identity" (see [9, 3.18]). It follows that the module $\mathrm{M}^{\otimes k}$ is isomorphic to $k$ iterations of restriction and induction (starting with the trivial module). This process works both for $\mathbf{S}_{n-1} \subseteq \mathbf{S}_{n}$ and $\mathbf{A}_{n-1} \subseteq \mathbf{A}_{n}$. In Figure 6 we see this amazing correspondence: the restriction and induction graphs for $\mathbf{A}_{3} \subseteq \mathbf{A}_{4}$ and $\mathbf{S}_{3} \subseteq \mathbf{S}_{4}$ correspond exactly to the representation graphs $\mathcal{R}_{\mathrm{V}}(\mathbf{T})$ and $\mathcal{R}_{\mathrm{V}}(\mathbf{O})$ and thus also to the affine Dynkin diagrams $\hat{E}_{6}$ and $\hat{E}_{7}$. In particular, this reveals why the partition algebras also work to describe the centralizer algebras $Z_{k}(\mathbf{T})$ and $Z_{k}(\mathbf{O})$ for $k$ odd as well as $k$ even. It remains an open question to fully understand the connections between $Z_{k}(\mathbf{I})$ and the generalized partition algebra End $_{\mathbf{A}_{5}}\left(\widetilde{\widetilde{M}}^{\otimes k}\right)$, where $\widetilde{\widetilde{M}}$ is the $\mathbf{A}_{5}$ module $\mathbf{A}_{5}^{(5)} \oplus \mathbf{A}_{5}^{(3,1,1)^{+}}$.

The irreducible representations of $\mathrm{P}_{k}(n)$ are labeled by integer partitions $\lambda \vdash n$ with $\left|\lambda^{\#}\right| \leq k$, where if $\lambda=\left(\lambda_{1}, \lambda_{2}, \ldots, \lambda_{\ell}\right)$ then $\lambda^{\#}=\left(\lambda_{2}, \ldots, \lambda_{\ell}\right)$. Using an inductive argument in [4], we give formulas for these dimensions which to our knowledge are new:

$$
\operatorname{dim} \mathrm{P}_{k}^{\lambda}(n)=\sum_{r=1}^{\ell}(-1)^{r-1} F_{r}^{\lambda}\left(\sum_{t=n-\lambda_{r}+r-1}^{n-2}\left(\begin{array}{c}
t \\
n-\lambda_{r}+r-1
\end{array}\right)\left\{\begin{array}{l}
k \\
t
\end{array}\right\}\right)+f^{\lambda}\left(\left\{\begin{array}{c}
k \\
n-1
\end{array}\right\}+\left\{\begin{array}{l}
k \\
n
\end{array}\right\}\right)
$$


where $f^{\lambda}$ is the number of standard tableaux of shape $\lambda,\left\{\begin{array}{c}k \\ j\end{array}\right\}$ is the Stirling number of the 2nd kind, and $F_{r}^{\lambda}$ is defined by: (1) $F_{1}^{\lambda}=f^{\lambda^{\#}}$, (2) $F_{r}^{\lambda}=0$, if $r>\lambda_{r}$, and (3) $F_{r}^{\lambda}=\sum_{\mu \subseteq \lambda, \mu_{r-1}=\mu_{r}=\lambda_{r}} F_{r-1}^{\mu} \cdot f^{\lambda / \mu}$, if $r>1$. We have analogous formulas in [4] for the partition algebras corresponding to $\mathbf{A}_{n}$.

\section{References}

[1] J. M. Barnes, G. Benkart, and T. Halverson. McKay centralizer algebras. Proc. London Math. Soc., 112(2):373-414, 2016.

[2] G. Benkart and T. Halverson. Exceptional McKay centralizer algebras. In preparation.

[3] G. Benkart and T. Halverson. Partition algebras $\mathrm{P}_{k}(n)$ with $n<2 k$. In preparation.

[4] G. Benkart and T. Halverson. Tensor power multiplicities for symmetric and alternating groups and dimensions of irreducible modules for partition algebras. In preparation.

[5] M. Bloss. The partition algebra as a centralizer algebra of the alternating group. Comm. Algebra, 33(7):2219-2229, 2005.

[6] C. W. Curtis and I. Reiner. Methods of representation theory. Vol. I and Vol. II. Pure and Applied Mathematics (New York). John Wiley \& Sons, Inc., New York, 1987.

[7] L. Gagnon and T. Halverson. Bases for McKay centralizer algebras. In preparation.

[8] F. M. Goodman, P. de la Harpe, and V. F. R. Jones. Coxeter graphs and towers of algebras, volume 14 of Mathematical Sciences Research Institute Publications. Springer-Verlag, New York, 1989.

[9] T. Halverson and A. Ram. Partition algebras. European J. Combin., 26(6):869-921, 2005.

[10] V. F. R. Jones. The Potts model and the symmetric group. In Subfactors (Kyuzeso, 1993), pages 259-267. World Sci. Publ., River Edge, NJ, 1994.

[11] P. Martin. Potts models and related problems in statistical mechanics, volume 5 of Series on Advances in Statistical Mechanics. World Scientific Publishing Co., Inc., Teaneck, NJ, 1991.

[12] P. Martin. The structure of the partition algebras. J. Algebra, 183(2):319-358, 1996.

[13] J. McKay. Graphs, singularities, and finite groups. In The Santa Cruz Conference on Finite Groups (Univ. California, Santa Cruz, Calif., 1979), volume 37 of Proc. Sympos. Pure Math., pages 183186. Amer. Math. Soc., Providence, R.I., 1980.

[14] N. J. A. Sloane. The on-line encyclopedia of integer sequences (OEIS).

[15] R. Steinberg. Finite subgroups of $\mathrm{SU}_{2}$, Dynkin diagrams and affine Coxeter elements. Pacific J. Math., 118(2):587-598, 1985.

[16] H. Wenzl. On tensor categories of Lie type $E_{N}, N \neq 9$. Adv. Math., 177(1):66-104, 2003. 